\title{
Context-Aware Service Front-Ends
}

\author{
Francisco Javier Caminero Gil \\ Telefonica I+D -Distrito C, \\ Edificio Oeste 1, Planta 5 \\ Ronda de la Comunicación, s/n \\ 28050 Madrid (Spain) - fjcg@tid.es
}

\author{
Fabio Paternò \\ CNR-ISTI, HIIS Laboratory \\ Via Moruzzi 156124 Pisa, Italy \\ fabio.paterno@isti.cnr.it
}

\author{
Vivian Genaro Motti \\ Université catholique de Louvain \\ Place des Doyens 1, 1348 \\ Louvain-la-Neuve Belgium \\ vivian.genaromotti@uclouvain.be
}

\begin{abstract}
Context-aware adaptation of user interfaces have been investigated since the early 80 's to provide mechanisms for stakeholders to propose, implement and execute adaptation, enabling users to efficiently interact with adaptive and adaptable applications. Today, adapting UIs according to the context of use becomes inevitable. Not only because users interact with applications from many distinct environments (platforms, devices and users' profile vary significantly), but also because such applications must provide a high usability level regardless of the contexts of use, efficiently adapting themselves according to the context. In this sense, Serenoa project proposes its $2^{\text {nd }}$ workshop, to join experts in the domain of context-aware adaptation to exchange experiences, discuss current trends, promote approaches, and raise awareness for this field.
\end{abstract}

\section{Author Keywords}

Context-Awareness; Service Front End; User Interface

Adaptation; Adaptivity; Adaptability.

\section{ACM Classification Keywords}

D2.2 [Software Engineering]: Design Tools and Techniques Modules and interfaces; user interfaces. D2.m [Software Engineering]: Miscellaneous - Rapid Prototyping; reusable software. H.5.1 [Information interfaces and presentation]: Multimedia Information Systems. H5.2 [Information interfaces and presentation]: User Interfaces - User-centered design.

\section{INTRODUCTION}

In a context in which users with different profiles interact by using different devices, modalities, platforms, systems, from distinct environments (concerning light, noise and stability level), it is neither scalable nor feasible for stakeholders to implement dedicated versions of interactive systems which provide high usability levels. Aiming to address this issue, the contextual information must be gathered and take into account in to adapt user interfaces (UIs), i.e. the content, navigation and presentation of interactive systems must be modified according to the context of use in which the user is located aiming to provide high usability levels. To support context-aware adaptation, several techniques, methods and strategies have been proposed in the last 30 years. Languages, frameworks, toolkits, architectural approaches and algorithms have been

Copyright is held by the author/owner(s). EICS'13, June 24-27, 2013, London, United Kingdom. ACM 978-1-4503-2138-9/13/06. continuously proposed, created and published since the early 80's. However, now, more than ever before, users interact from contexts that significantly vary, and considering such variations to appropriately adapt applications becomes a great challenge. In this context, Serenoa project proposes its second workshop. Named CASFE'2013, Context-Aware Services Front End, this workshop is dedicated to join experts in this field to discuss, comment, review, publish and analyze the latest achievements, current issues, and future trends for adaptation.

\section{WORKSHOP}

Several works attempted to address the challenge of context-aware adaptation including valuable inputs from various disciplines, such as: ubiquitous computing, pervasive applications, ambient intelligence, artificial intelligence, engineering interactive computing systems, mobile human-computer interaction. These contributions are often expressed in a format that prevents them to be compared with others, mainly because they are heterogeneous and their format is inconsistent. This workshop is aimed at addressing context-aware adaptation of user interfaces via an interaction model according to three dimensions (adapted from [1]): a descriptive power that consists in characterizing a sufficiently large spectrum of adaptation techniques according to a unified format, an evaluative power that consists in comparing different adaptation techniques based on the same format, and a generative virtue that is intended to identify holes in the resulting design space to foster further research and development of new interaction techniques. "A good interaction model must strike a balance between generality (for descriptive power), concreteness (for evaluative power) and openness (for generative power)" [1]. This means that there is a need for creating a design space for context-aware adaptation of any kind of user interface that should exhibit enough expressiveness (for guarantying enough descriptive power), decidability (for ensuring the ability to assess any adaptation technique), and flexibility (for accommodating new adaption techniques that were previously unforeseen).

There is also a need for discussing and reviewing the state of the art in the domain of UI adaptation under the viewpoint of context-awareness since most surveys are either obsolete [2] or do not address context-awareness [3]. This state of the art will be namely focusing on models, methods, 
and tools that support context-aware adaptation of UIs, in particular in order to address existing challenges [4].

Aims and Goals. This workshop is targeted at industrial and academic participants, with interest in the adaptation field, belonging to different domains of expertize, as: computer scientists, engineers, architects and psychologists.

Format. The workshop will last half day dedicated to discuss and review the state of the art in the area of contextaware adaptation: criteria, requirements, design spaces, rules, techniques, tools and languages for context-aware adaptation of user interfaces. Selected papers will be presented, and discussed, resulting into an updated and accurate version of the workshop material. A projector will be used.

Participants. Participants are welcome from any background or discipline that is concerned by contextaware adaptation ranging from psychology, social sciences to computer science. The expected amount of participants will be around 15, but could be extended depending on the interest of the topic.

\section{ORGANIZERS}

Francisco Javier Caminero Gil is the project coordinator of the FP7-ICT5-Serenoa (Multidimensional context-aware adaptation of Service Front-ends) project funded by the European Commission.

Fabio Paternò is research director of the Laboratory on Human Interfaces in Information Systems at ISTI, CNR in Pisa, Italy. He has been Co-Chair of the ACM CHI 2000, INTERACT 2003 and 2005, and chair of MobileHCI'2002, EICS'2011 and AmI'2012.

Vivian Genaro Motti is a $\mathrm{PhD}$ candidate at Université catholique de Louvain where she is a member of the Louvain Interaction Laboratory (LILab). She works as a research assistant for FP7-ICT5-Serenoa project and has publications on ICWE, EICS, AVI, Interact, and WWW.

\section{POTENTIAL OUTCOMES}

We intend to discuss at the workshop the following topics:

- What are the major challenges (e.g., conceptual, methodological, technical, organizational) for developing context-aware adaptation of user interfaces?

- For which kinds of systems or applications are contextaware user interfaces particularly useful?

- When and how could we measure the effectiveness, the efficiency of context-aware adaptation?

- How could we measure the quality of the user interface resulting from a context-aware adaptation process?
- In which ways will context-aware adaptation affect user interfaces in the future and how will they evolve?

- What kinds of context-aware adaptation do you see as particularly promising?

\section{CALL FOR PAPERS}

We will accept participants based on the paper quality and the diversity of their backgrounds, aiming at an interdisciplinary group. The authors of accepted papers will be asked to provide a refined version of their paper one month before the workshop based on a preliminary version of workshop material: a design space, an adaptation specification language, some example of adaptation rules. These position papers will be circulated in advance for participants to get an understanding of the mutual views and to provide a starting point for the discussion. The papers should be submitted via EasyChair.

\section{CONCLUSION}

Although there is a great motivation for the topic of interest of this workshop, there are still many open issues and a lot of room from improvement. That is why we propose a second release of Serenoa workshop. To bring together experts in this domain, promote discussions, exchange of experiences and raise awareness for this relevant topic.

\section{ACKNOWLEDGMENTS}

This workshop is supported by Serenoa, funded by the European Commission's Seventh Framework Programme under grant agreement $\mathrm{n}^{\mathrm{o}} 258030$ (FP7-ICT-2009-5).

\section{REFERENCES}

1. Beaudouin-Lafon, M. Designing interaction, not interfaces. In: Proc. of ACM Working Conf. on Advanced Visual Inter-faces AVI'2004 (Gallipoli, May 25-28, 2004). ACM Press, New York (2004), pp. 15-22.

2. Dieterich, H., Malinowski, U., Kuhme, T., and Schneider-Hufschmidt, M. State of the art in adaptive user interfaces. In: Schneider-Hufschmidt, M., Kuhme, T., Malinowski, U. (Eds.), Adaptive User Interfaces Principles and Practice. Elsevier Science Publishers B.V., Amsterdam (1993), pp. 13-48.

3. López-Jaquero, V., Vanderdonckt, J., Montero, F., and González, P. Towards an Extended Model of User Interface Adaptation: the ISATINE framework. In: Proc. Of EIS'2007 (Salamanca, 22-24 March 2007), J. Gulliksen, M.B. Harning, Ph. Palanque (Eds.). LNCS, Vol. 4940. Springer-Verlag, Berlin (2008), pp. 374-392.

4. Vanderdonckt, J. Model-Driven Engineering of User Interfaces: Promises, Successes, and Failures. In: Proc. of 5 th Annual Romanian Conference on HumanComputer Interaction ROCHI'2008 (Iasi, September 1819, 2008). S. Buraga, I. Juvina (Eds.). Matrix ROM, Bucharest (2008), pp. 1-10. 